\title{
Reconfigurable Origami Antennas: A Review of the Existing Technology and its Future Prospects
}

\author{
Hafiz Suliman Munawar \\ University of New South Wales (UNSW) Sydney, Australia \\ Email address: h.munawar@unsw.edu.au
}

Received: 01 January 2020; Accepted: 08 March 2020; Published: 08 August 2020

\begin{abstract}
Owing to the much-gained popularity and unique properties of origami antenna, a review of some of the important types of these antennas is conducted. Origami is an ancient paper folding technique which has many applications in technical and non-technical fields. With the growing trend of utilizing origami concept, it was integrated with the electromagnetic field to realize reconfigurable antennas. This review contains some of the important applications in which the origami antennas are widely used to make the system more efficient. The development of the antennas over the years and some state-of-the-art designs are discussed along with the future trends in design and manufacturing of origami antennas. Some unique abilities of origami antennas which make them better choice than other antennas are smaller size and volume in addition to being light weight, cost efficient and easily manufacturing. They are widely used to reduce the size of bulky antennas which is very handy in many space and air borne facilities and provide reconfigurable frequency. They are even used to provide mobile range in far off regions which are not accessible with the use of ordinary antennas. The field of antenna design is vast and provides a lot of room for further advancements which are covered in future trends of the paper. This paper provides a detailed review of the different designs of antenna and points towards possible future enhancements and state of the art designs for the fabrication of antenna.
\end{abstract}

Index Terms: Reconfigurable antennas, origami antennas, deployable, multifunctional, flexible antennas

\section{Introduction}

Origami is an ancient art of paper folding whose concept is being used in various applications of robotics, architecture and medical devices. For the past two decades the concept of folding and unfolding systems have been used by researchers and mathematicians [1]. The idea of origami has been used in telescopes lens which are carried into orbits by space shuttles, heart stent that is sent through the blood stream and unfolds at desired location and many space applications which require miniaturized structures because of small spaces. Origami concept has been used with integration of electromagnetic theory for the realization of reconfigurable antennas [2]. Origami antennas gained popularity because of their ability to reconfigure mechanically by changing their physical geometry that changes their electromagnetic properties. They can transform 2D structures into a range of $2 \mathrm{D}$ and $3 \mathrm{D}$ shapes by using rigid foldability mechanisms. Various performance parameters such as beamforming and scanning range can be controlled by changing the antenna's shape or element separation [3].

These antennas are multifunctional and have the potential to develop new electromagnetic systems with various transformational abilities. Typical origami antennas are made by using paper as antenna substrate and conductive ink as metal parts till now, but they are not resistant under heavy mechanical stresses. Many durable and robust techniques have been developed over the years which are being extensively used in E- textile [4]. Height of antenna is controlled by various operations of folding and unfolding to obtain reconfigurable characteristics which include the use of servo motor in space borne applications or by pressing the spring base [5]. Origami antennas provide the advantage of reconfigurable frequency by changing the physical geometry of the antenna. Yagi loop antennas use the origami spring base in many applications because of its ability to minimize the size with provision of high gain and front to back ratio [6]. Advantages of using origami antennas include easy and fast designing procedures, lesser volume and cost saving. On the other hand, they have drawbacks of less durability and lack of materials for robust actuation [5]. Improvements and advancements are needed in this field to overcome these issues which are discussed in the later section of this paper. This paper provides significant insight in the development of origami antennas with its latest technologies along with some future trends. Some of the existing origami antennas and their applications are reviewed to understand their basics and have clear knowledge of their uses. 
This review paper is organized into sections. Section II discusses some of the existing techniques used in manufacturing origami antennas. Section III covers their applications and uses. Section IV contains the development of these antennas in past and present. Lastly, section $\mathrm{V}$ concludes the paper with some future trends of origami antennas.

\section{Categorization and Methodology}

The concept of origami is ancient which is used in various applications but its use in electromagnetism is new and very important. Many antennas are designed on this concept and to achieve the best possible results, different folding and unfolding techniques are found in the literature.

As the concept of origami antennas is vast and has numerous applications in different fields of research, so it is needed to set the boundary and scope of this review. This review contains papers published in the conferences and journals from International Electrical and Electronics Engineers (IEEE) in last 5 years which cover the topics related to origami antennas and their applications.

This section will discuss some of the origami antenna designs which are manufactured for special purpose and provide different facilities. The purpose of reviewing the designs is to analyze their applications and extract information about how the technology can be used further for enhancing their usability.

\subsection{Origami micro strip antenna}

In many wireless devices, two-dimensional pattern techniques are used for fabrication of radiating elements which include photolithography, stamping and milling. In some applications, 2D substrates are transformed into 3D structures using the response from the light which is also called self-folding [7]. For the manufacturing, a copper foil is supported by pre- strained polystyrene sheets which contain black ink and it actively absorbs the light controlling the shape of transform.

An antenna with similar working principle was designed by Hayes et al [7] which had the ability to transform a monopole antenna into a micro strip transmission line and micro strip patch antenna that transforms into monopole antenna within seconds. These antennas have advantages in wireless communication as they are self-folding and produce hands free response upon exposure to light.

\subsection{Origami spring antenna}

Foldable antennas are more useful than other antennas because they can easily be expanded and collapsed according to the use and save a lot of space. These collapsible antennas are reconfigurable and possess unique capabilities with enhanced performance.

In [8], Yao et al gave design of a foldable antenna whose operating frequency was controlled by its height. The proposed design was folded by a dielectric substance which collapses when the pressure is applied on the spring and deploys when the pressure is removed. This antenna had the unique ability of providing different heights which made it operate in different frequencies and gain. Results from the spring-based structure proved that the height can precisely be controlled by it and provide various working frequencies.

\subsection{Tetrahedron origami antenna}

High gain is one of the most important parameters of any antenna and to obtain it various techniques and enhancements are made. Different methods by which high gain antennas are obtained include the use of metal-stacked rings, Complementary Electromagnetic band-gap (EBG) substrate integrated waveguide (SIW) and artificial magnetic conductors [5].

To obtain a high gain origami antenna, a novel design was presented by Shah et al in [4], which had a tetrahedron geometry. Antenna consisted of a triangular shaped monopole antenna, reflector and two parasitic strips on a paper substrate which was cost effective and small. Gain was increased using reflectors, directors and folding procedure. Results of the fabricated design showed a much wider bandwidth along with high gain value of $9.5 \mathrm{dBi}$ at $2.6 \mathrm{GHz}$.

\subsection{Origami Yagi loop antenna}

Yagi antennas have vast uses in radio and point to point communication as they provide high gain and simple structures. Some of the Yagi antennas operating at low frequency have bulky structures for which origami antennas are integrated to reduce the size of the antenna. Advantage of origami spring is that by applying pressure at any level controls the whole body.

Considering the use of using these antennas in integration, Yao et al presented a design in [6], which can compress the length of the Yagi loop by $5 \mathrm{~cm}$ using a servo motor and reduced $70 \%$ of the volume.

Antenna results showed high directional gain and front to back ratio.

\subsection{Origami accordion antenna}

Height changing origami antennas are widely used because of their ease in operation and manufacture. For this reason, another antenna was proposed by Yao et al which possess height of $160 \mathrm{~mm}$ when unfolded and $40 \mathrm{~mm}$ when 
completely folded [2]. Height of the antenna was easily controlled by the telescoping mechanism and provided high gain and reconfigurable operating frequency.

\subsection{Tri-band reconfigurable origami antenna}

For obtaining frequency reconfigurable antennas various methods were employed in addition to these, a new method was proposed by Liu et al which had a simple feeding mechanism with circular polarization. The antenna was a helical array which operated in three states providing three operating frequencies connected by one common reflector. High gain values of $2.07 \mathrm{GHz}, 3 \mathrm{GHz}$ and $4.45 \mathrm{GHz}$ were obtained for respective operating states [9].

For clear understanding of different types of origami antennas, Table 1 contains a comparative analysis of them. Understanding the advantages of different types of antenna helps in analyzing their applications and possibly bringing in newer applications.

Table 1 Comparative Analysis of Some origami antennas.

\begin{tabular}{|c|c|c|}
\hline Types of Antenna & Advantages & Applications \\
\hline Origami micro strip antenna & Self-folding, hands free. & Simple light activated switches \\
\hline Origami spring antenna & Precise control by the spring base, Directional. & Communication applications \\
\hline Tetrahedron origami antenna & $\begin{array}{l}\text { Wide impedance bandwidth, Low cost, Easy } \\
\text { manufacturing. }\end{array}$ & $\begin{array}{l}\text { Point to point wireless communication, } \\
\text { Outdoor applications. }\end{array}$ \\
\hline Origami Yagi loop antenna & $\begin{array}{l}\text { Whole length can be controlled by servo gripper, } \\
\text { High gain and front to back ratio. }\end{array}$ & Space borne structures and satellites \\
\hline Origami accordion antenna & $\begin{array}{l}\text { Maximum gain by simple telescoping } \\
\text { mechanism. }\end{array}$ & Space borne structures and payloads \\
\hline $\begin{array}{l}\text { Tri-band reconfigurable origami } \\
\text { antenna }\end{array}$ & $\begin{array}{l}\text { Simplified feeding port, improved gain as } \\
\text { compared to the single element. }\end{array}$ & Satellite radio, WiMax. \\
\hline
\end{tabular}

\section{Application and uses}

Origami antennas have applications in many useful products because of their smaller and adjustable size along with good gain values. They are lighter in weight and possess frequency reconfigurable properties. This section discusses some of the important applications and uses of origami antennas in different fields.

\subsection{E-textile Embroidery}

Wearable antennas have gained much popularity over the years and are still developing for further use. One of the modes of wearable antennas uses the textile and renders very useful. Keeping the view that origami antennas can easily be integrated into textile antennas because of their small size, Chaudhari et al developed an origami antenna using the E-threads. A new class of reconfigurable origami antennas was presented which was not only robust and durable but also flexible. Automated embroidery with silver coated threads were used to realize conductive surfaces. This proposed antenna paved way for the use of origami antennas in E-textile antennas and can easily be used for the application in wearable antennas [4].

\subsection{Extended mobile range}

Reliable long-range communication is needed by civilians as well as military which is made possible using reconfigurable and deployable antennas.

One shortcoming of these antennas is their size and many antennas have been developed to address this issue, Carrara et al presented a reconfigurable origami antenna design in [10], which operated in extended mobile frequency range. This antenna was designed to overcome the bulky size of the Yagi antenna extending the frequency band to $806 \mathrm{MHz}$ from $698 \mathrm{MHz}$ which enabled the remote areas to gain access to mobile communications.

\subsection{Military}

Most of the communication in military is done by using walkie-talkies, but they can be tapped easily, so satellite communication is used for conveying secret messages. Antennas used for satellite communication are big in size and needs to be transported with special arrangement. In order to solve this issue, Shah et al developed a light weight, durable origami antennas which had origami tetrahedron structures with paper substrate and provided high gain in addition to low cost and easy manufacturing [11].

\subsection{Space applications}

Due to the size reducing ability of origami antennas, they are used extensively in space satellite because they have small space for the hardware installments. Also, the structure of antennas in satellite applications must be light weight and deployable so origami antennas are the best choice for these applications because they possess these qualities and provide good performance in terms of gain [12]. 


\subsection{Disaster Management}

These antennas prove to be very useful for outdoor applications as they have reasonable strength and can endure extreme weather conditions like wind and rain easily. The use of antennas is also applicable in terms of evacuation of large populations from a disaster or calamity struck area [13]. Gaining signals remotely from areas hit by floods or earthquakes can provide an estimate of the number of people stuck there through satellite communication with the rescue teams. Moreover, medical aid can be provided on the site of disaster by using antennas to enhancing telemedicine and emergency car $[14,15]$.

\section{Origami Antennas Development over the Years}

Designing of multifunctional deployable antennas require integration of origami math, mechanics and electromagnetism. First designs of the antennas using origami concept assumed zero thickness or very thin substrates that showed good performance in terms of polarization and radiation pattern as compared to typical antennas. Primitive antennas lacked in durability and robustness because after a small number of folds, the antenna substrates started cracking especially at the places of relatively high stress.

Extreme weather conditions like rain and wind also affected the antenna structures and performance. In order to resolve the physical issues of the antennas, use of durable materials were needed which endured the multiple folding and unfolding along with the use of thick and rigid substrates [3].

To tackle these problems, new antennas were designed which were reconfigurable, durable and foldable and used in various applications like space and air borne systems [3]. Recently Yao et al presented the antenna design which reduces the stresses of structure and easily transforms a dipole antenna to a conical structure by use of flasher model [8]. Another design of using a thick origami antenna was presented that enables the elements to fold fully. A recent design published by Rohde et al provided the design of light weight, multi-functional antenna that provided high gain value by solving the impedance matching problems [16].

Though many developments and advancements have been made in this field and it is at a stage where it has various applications, still it needs a lot of attention and research to find even efficient and better ways to manufacture reconfigurable origami antennas.

\section{Conclusion and Future Trends}

The ancient Chinese art of paper folding is used in many Origami antennas gained much importance in various fields of technology and science. In recent years, origami techniques were integrated with electromagnetism to use them in realization of reconfigurable antennas. Origami antennas became much popular because of their unique ability of reducing the size of bulky antennas which makes them useful in many applications. They are light weight, easily manufactured, cost efficient and give better gain values. The ability to reconfigure frequency by changing the physical geometry of the antenna is an important aspect of these antennas which is done by changing height of the antenna or by using a spring.

In order to understand working and importance of different types of origami antennas this review is done which discusses some of the important reconfigurable origami antennas. Applications of these antennas in some of the important fields are discussed along with brief understanding of their mode of operation. Development of origami antennas over the course of years in addition to some of the latest techniques which are introduced to make an even better product are contained in this paper. Though many advancements have been made in last 5 years for making even efficient antennas, it still needs exploration and research.

Some of the expected future trends in this field include the work toward maintaining the robustness of antennas while they morph to achieve different electromagnetic operations. Newer mechanisms will be developed to enhance the capabilities of the antennas. For complete and appropriate knowledge of the origami electromagnetic designs, unified computational tools will be deployed which include multi-physics, origami math and multi-scale modeling algorithms. This review has provided a picture of the existing work in the field and has pointed towards ways in which possible future enhancements can be made. The comparison of different types of antennas and their application has done to clarity the roles being played by different types of antennas. Researchers working in this area can further enhance and upgrade the design and capacity of these antennas to harness more usefulness out of this technology in the future.

\section{References}

[1] Shah, S.I.H., Tentzeris, M.M. and Lim, S., 2017. Low-cost circularly polarized origami antenna. IEEE Antennas and Wireless Propagation Letters, 16, pp.2026-2029.

[2] Yao, S., Georgakopoulos, S.V., Cook, B. and Tentzeris, M., 2014, June. A novel reconfigurable origami accordion antenna. In 2014 IEEE MTT-S International Microwave Symposium (IMS2014) (pp. 1-4). IEEE.

[3] Georgakopoulos, S.V., 2019, April. Reconfigurable Origami Antennas. In 2019 International Applied Computational Electromagnetics Society Symposium (ACES) (pp. 1-2). IEEE. 
[4] Chaudhari, S., Alharbi, S., Zou, C., Shah, H., Harne, R.L. and Kiourti, A., 2018, July. A New Class of Reconfigurable Origami Antennas Based on E-Textile Embroidery. In 2018 IEEE International Symposium on Antennas and Propagation \& USNC/URSI National Radio Science Meeting (pp. 183-184). IEEE.

[5] Shah, S.I.H., Lee, D., Tentzeris, M.M. and Lim, S., 2016. A novel high gain tetrahedron origami. IEEE Antennas and Wireless Propagation Letters, 16, pp.848-851.

[6] Yao, S., Liu, X., Gibson, J. and Georgakopoulos, S.V., 2015, July. Deployable origami Yagi loop antenna. In 2015 IEEE International Symposium on Antennas and Propagation \& USNC/URSI National Radio Science Meeting (pp. 2215-2216). IEEE.

[7] Hayes, G.J., Liu, Y., Genzer, J., Lazzi, G. and Dickey, M.D., 2014. Self-folding origami microstrip antennas. IEEE Transactions on Antennas and Propagation, 62(10), pp.5416-5419.

[8] Yao, S., Liu, X., Gibson, J. and Georgakopoulos, S.V., 2015, July. Deployable origami Yagi loop antenna. In 2015 IEEE International Symposium on Antennas and Propagation \& USNC/URSI National Radio Science Meeting (pp. 2215-2216). IEEE.

[9] Liu, X., Yao, S., Russo, N. and Georgakopoulos, S., 2018, July. Tri-band Reconfigurable Origami Helical Array. In 2018 IEEE International Symposium on Antennas and Propagation \& USNC/URSI National Radio Science Meeting (pp. 1231-1232). IEEE.

[10] Carrara, G.P., Russo, N.E., Zekios, C.L. and Georgakopoulos, S.V., 2019, July. A Deployable and Reconfigurable Origami Antenna for Extended Mobile Range. In 2019 IEEE International Symposium on Antennas and Propagation and USNC-URSI Radio Science Meeting (pp. 453-454). IEEE.

[11] Shah, S.I.H., Lim, S. and Tentzeris, M.M., 2017, March. Military field deployable antenna using origami. In 2017 International Workshop on Antenna Technology: Small Antennas, Innovative Structures, and Applications (iWAT) (pp. 72-73). IEEE.

[12] Rohde, D., Noghanian, S., Chang, Y.H. and Sharma, S.K., 2019, July. Two Element Series Fed Origami Antenna. In 2019 IEEE International Symposium on Antennas and Propagation and USNC-URSI Radio Science Meeting (pp. 1111-1112). IEEE.

[13] Munawar, H.S., Hammad, A., Ullah, F. and Ali, T.H., After the Flood: A Novel Application of Image Processing and Machine Learning for Post-Flood Disaster Management.

[14] Munawar, H.S., Awan, A.A., Maqsood, A. and Khalid, U., REINVENTING RADIOLOGY IN MODERN ERA.

[15] Munawar, H.S., AAwan, A.A., Khalid, U., Munawar, S. and Maqsood, A., 2017. Revolutionizing Telemedicine by Instilling H. 265. International Journal of Image, Graphics \& Signal Processing, 9(5).

[16] Seiler, S.R., Bazzan, G., Fuchi, K., Alanyak, E.J., Gilman, A.S., Alexander, G.W., Cook, A., Buskohl, P.R., Pallampati, S., Espinal, F.A. and Sessions, D., 2018, July. An Origami Inspired Circularly-Polarized Folding Patch Antenna Array. In 2018 IEEE International Symposium on Antennas and Propagation \& USNC/URSI National Radio Science Meeting (pp. 181-182). IEEE.

\section{Author's Profile}

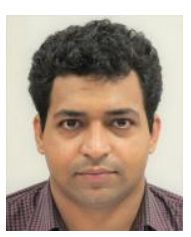

Hafiz Suliman Munawar is a PhD student at the University of New South Wales (UNSW), Australia. He is a multi-disciplinary researcher with experience in machine learning, disaster management and artificial intelligence. Hafiz has several international publications in various journals and conferences and has actively been working on disaster management using machine learning.

How to cite this paper: Hafiz Suliman Munawar, " Reconfigurable Origami Antennas: A Review of the Existing Technology and its Future Prospects ", International Journal of Wireless and Microwave Technologies(IJWMT), Vol.10, No.4, pp. 34-38, 2020.DOI: 10.5815/ijwmt.2020.04.04 